Tohoku J. Exp. Med., 2007, 213, 121-127

\title{
Identifying Medical Interview Behaviors that Best Elicit Information from Patients in Clinical Practice
}

\author{
Yousuke Takemura, ${ }^{1}$ Reiko Atsumi ${ }^{1}$ and Tsukasa Tsuda ${ }^{1}$ \\ ${ }^{1}$ Department of Family Medicine, Mie University School of Medicine, Mie, Japan
}

Takemura, Y., Atsumi, R. and Tsuda, T. Identifying Medical Interview Behaviors that Best Elicit Information from Patients in Clinical Practice. Tohoku J. Exp. Med., 2007, 213 (2), 121-127 — Many textbooks indicate the desirability of using specific medical interview behaviors to obtain information from patients, although little evidence has been presented. The aim of this study is to investigate the relationships between the use of medical interview behaviors and the amount of information obtained. The behaviors studied included: open-ended questions, facilitation (comments or interviewer behavior that encourages the patient to continue talking), the open-to-closed cone (the gradual narrowing of focus from an initial nondirective approach to a more direct exploration), summarization (providing the patient with an explicit verbal summary of the information gathered thus far), and surveying problems. The subjects were 315 patients who visited an outpatient facility. These medical interviews were videotaped and reviewed by a trained rater using the Takemura Medical Interview Rating Scale, which was developed to assess the use of particular medical interview behaviors and to measure the amount of information obtained from patients regarding their chief physical complaints. Significant positive relationships were found between three particular interview behaviors and the amount of information obtained: facilitation, the open-to-closed cone, and summarization. These positive relationships were still present after adjusting for other medical interview behaviors used, and after adjusting for the time duration of the medical interviews $(F=15.3, p<0.0001 ; F=$ $40.1, p<0.0001 ; F=5.57, p=0.019$, respectedly). This study reveals a positive relationship between three specific medical interview behaviors (facilitation, the open-to-closed cone, and summarization) and the amount of information obtained in a real clinical practice setting. — interviews; information; data collection; clinical competence

(C) 2007 Tohoku University Medical Press

Certain medical interview behaviors are thought to be effective for gathering medical information from patients. Several reports have shown that the medical history obtained during the medical interview provides $60-80 \%$ of the information needed for an accurate diagnosis (Hampton et al. 1975; Sandler 1980; Kassirer 1983; Peterson 1992). Numerous medical inter- view behaviors can be used to gather medical information from patients, including open-ended questions (Rutter and Cox 1981; Cox et al. 1981a, b; Beckman and Frankel 1984; Roter and Hall 1987; Maguire et al. 1996; Marvel et al. 1999; Takemura et al. 2004), facilitation (Rogers 1980; Egan 1990; Wissow 1994), the open-to-closed cone (Cox et al. 1981a; Bird and Cohen-Cole

Received May 8, 2007; revision accepted for publication August 13, 2007.

Correspondence: Yousuke Takemura, Department of Family Medicine, Mie University School of Medicine,

2-174 Edobashi, Tsu, Mie 514-8507, Japan.

e-mail: yousuke@clin.medic.mie-u.ac.jp 
1990), summarization (Maguire et al. 1996), and surveying problems (White et al. 1994; Barrier et al. 2003). These various behaviors have been explained in many articles or medical interview textbooks (Bird and Cohen-Cole 1990; Lipkins et al. 1995; Participants in the Bayer-Fitzer Conference on Physician-Patient Communication in Medical Education 2001). Open-ended questions are designed to allow patients to describe their symptoms using their own vocabulary and personal experiences. Although physicians are encouraged to use open-ended questions early in the medical interview, they can be used at any time. Facilitation includes comments or interviewer behavior that encourages the patient to continue talking along the same lines. For example, nodding without interrupting the patient is a type of facilitation. The open-to-closed cone is the gradual narrowing of focus from an initial nondirective approach to a more direct exploration of a specific diagnostic hypothesis. Summarization involves providing the patient with an explicit verbal summary of the information gathered thus far. It allows the patient to confirm that the physician has understood what he or she has said, and it also acts as a facilitative opening, allowing the patient to further explain his or her problems and thoughts. Surveying problems is an often overlooked medical interview behavior, where the physician attempts to briefly scan the full range of a patient's problems, such as by asking, "What else is bothering you?".

Most studies of medical interview behaviors used a relatively small number of subjects, and many studies failed to control for significant confounders, such as the time duration of the medical interview (Beisecker 1989; Wilson and Child 2002; Ishikawa et al. 2005). Some studies used standardized patients, rather than real patients in a clinical practice. The aim of the present study was to investigate the relationships between the use of several medical interview behaviors and the amount of information obtained from patients during the medical interview, using a relatively large sample size, in a real clinical practice setting. Because time duration can significantly affect the amount of information obtained, an adjustment was made in this study to eliminate its effects.

\section{Subjects And Methods}

Subjects

The subjects were 315 patients (163 female and 152 male) who visited the outpatient facility of the Department of Family Medicine at Mie University Hospital, in Mie, Japan, as new patients from September 2004 to May 2005. The subjects were all over age 15 years and mainly had common diseases like hypertension, hypercholesterolemia, diabetes, depression, and so on. The patients had a wide range of ages and social classes, and they came from both rural and urban areas. They were interviewed by 43 fifth-year medical students (15 female and 28 male) who were doing their family medicine clinical clerkships, nine family medicine residents (4 female and 5 male), and two male attending physicians from the Department of Family Medicine. The medical interviews were videotaped. The videotapes were later reviewed and rated by a trained staff member (R.A.) using the Takemura Medical Interview Rating Scale (TMIRS). The time duration of each medical interview was measured using the videotape. This study was approved by the Research Ethics Committee of the Mie University School of Medicine.

\section{Measurements}

There are several published methods used to objectively assess the medical interview. The Roter Interaction Analysis System (RIAS) is one widely-used tool (Roter 1991). However, the RIAS includes only a limited number of medical interview behaviors, and it does not include facilitation, the open-to-closed cone, summarization, or surveying problems, all of which we wanted to investigate. Therefore the authors developed the TMIRS to assess the use of specific medical interview behaviors and to measure the amount of information obtained from patients during the medical interview. Some systems of the RIAS were used to construct the TMIRS.

To construct the TMIRS, first we reviewed medical interview textbooks to identify medical interview behaviors (Bird and Cohen-Cole 1990). We then invited five Japanese experts in the teaching of medical interview behaviors and patient-physician communication to join the item development group, including three family physicians, a general internist, and a behavioral scientist. The experts discussed the items of the rating scale, suggested additional items, and compared the items identi- 
fied during their qualitative work with those previously identified.

Using this qualitative process, we selected openended questions, facilitation, the open-to-closed cone, summarization, and surveying problems as the medical interview behaviors to be studied. Open-ended questions, summarization, and surveying were measured on a two-point scale (used, not used), since the aim of this investigation was to know the effect of using or not using these behaviors on the amount of information obtained. Facilitation and the open-to-closed cone were measured on a three-point scale (frequently used, moderately used, not used), because in previous studies many interviewers used these behaviors, and we wanted to assess the extent to which they did so. We used the number of backchannel responses, as defined by the RIAS, to categorize the use of facilitation into three levels (Roter 1991). In the RIAS, the back-channel is an indicator of sustained interest, attentive listening, or encouragement emitted by an interviewer when he or she does not hold the speaking floor. If an open-ended question was used and then closed-ended questions were used to clarify the contents of the symptoms elicited by the open-ended question later, the open-to-closed cone was defined to be used. Furthermore, if the information was elicited although the interviewer didn't use open-ended question, for example by facilitation behavior, and then closed-ended questions were used to get the contents of the symptoms elicited, we also counted it as the open-to-closed cone was used in this study.

The amount of information obtained during the interview was measured using nine aspects of the chief physical complaint: duration, frequency, onset of symptoms, location, quality, severity, context (time or place when the symptoms occurred), modifying factors, and associated symptoms. These items were selected by a review of medical interview textbooks and through discussions by the five experts. These items of information were measured using a two-point scale (obtained, not obtained). The total amount of information obtained from the patient was then calculated as below:

$$
\begin{gathered}
\text { Information = duration + frequency + onset }+ \\
\text { location + quality + severity + context }+ \\
\text { modifying factors + associated symptoms }
\end{gathered}
$$

The TMIRS was assessed for its validity and reliability as follows. Face validity was examined by the expert group using the Delphi method. Test-retest reliability was then examined for each item of the medical interview rating scale, using 95 videotaped medical inter- views. For this purpose, the rater re-examined the same videotape of the medical interview one week after the first evaluation. The agreement for each scored item (open-ended questions, facilitation, the open-to-closed cone, summarization, and surveying problems) was investigated, and reliability measurements (Cohen's kappa coefficient) were 0.98 to 1.00 . The kappa coefficients for each item of medical information (duration, frequency, onset, location, quality, severity, context, modifying factors, and associated symptoms) ranged between 0.86 and 1.00. The kappa coefficient for the calculated amount of information obtained was 0.87 .

\section{Statistical analyses}

Data from a total of 314 medical interviews were eligible for analysis. One medical interview was excluded due to a mechanical problem with the videotape recording. The relationship between each medical interview behavior and the amount of information was analyzed by the ANOVA method. For medical interview behaviors measured on a three-point scale, a test for trend was calculated to assess the dose-response relationship. To control for confounding factors, including the time duration of the medical interview and the other medical interview behaviors used, we used the GLM procedure, which uses the method of least squares to fit general linear models. All data were stored and analyzed on a computer using SAS/STAT version 9.1 software (SAS Institute, Inc. 2004).

\section{RESUlts}

The mean (standard deviation [s.D.]) time duration of the medical interviews was 19.9 (11.5) min. The mean (s.D.) information score was 7.2 (1.6) on a scale from 0 to 9.

Table 1 shows the medical interview behaviors used for gathering information from patients. We found that facilitation, open-ended questions, and surveying were often used, while summarization was used relatively less.

Table 2 shows the items of information gathered during the medical interviews. The duration of symptoms of the chief physical complaint was obtained relatively less frequently than other items of information.

Table 3 shows the relationships between the medical interview behaviors used and the amount of information obtained. There were significant 
TABLE 1. Medical interview behaviors used for gathering medical information from patients.

\begin{tabular}{lccc}
\hline \multicolumn{1}{c}{ Behavior } & Frequently used (\%) & Moderately used (\%) & Not used (\%) \\
\hline Facilitation & $125(39.8)$ & $167(53.2)$ & $22(7.0)$ \\
Open-to-closed cone & $208(66.2)$ & $100(31.9)$ & $6(1.9)$ \\
\hline \multicolumn{1}{c}{ Behavior } & Used (\%) & Not used (\%) & \\
\hline Open-ended question & $220(70.1)$ & $94(29.9)$ \\
Summarization & $120(38.2)$ & $194(61.8)$ \\
Surveying problems & $198(63.1)$ & $116(36.9)$ \\
\hline
\end{tabular}

TABLE 2. Items of medical information gathered during the medical interviews.

\begin{tabular}{lcc}
\hline & Obtained $(\%)$ & Not obtained $(\%)$ \\
\hline Duration & $143(45.5)$ & $171(54.5)$ \\
Frequency & $237(75.5)$ & $77(24.5)$ \\
Onset & $308(98.1)$ & $6(1.9)$ \\
Location & $310(98.7)$ & $4(1.3)$ \\
Quality & $273(86.9)$ & $41(13.1)$ \\
Intensity & $216(68.8)$ & $98(31.2)$ \\
Context & $257(81.8)$ & $57(18.2)$ \\
Modifying factors & $210(66.9)$ & $104(33.1)$ \\
Associated symptoms & $296(94.2)$ & $18(5.8)$ \\
\hline Information* & \multicolumn{3}{c}{$7.17 \pm 1.62^{* *}$} \\
\hline
\end{tabular}

${ }^{*}$ Information: duration + frequency + onset + location + quality + severity + context

+ modifying factors + associated symptoms.

${ }^{* *}$ Mean \pm S.D.

positive relationships between facilitation, the open-to-closed cone, and summarization and the amount of information obtained. The amount of information increased with increases in the use of facilitation and the open-to-closed cone.

We found a significant relationship between the duration of the medical interview and the amount of information obtained $(t=4.53, p<$ $0.0001)$. This positive relationship was still present after adjusting for the time duration of the medical interview, and after adjusting for the other medical interview behaviors used.

\section{Discussion}

Many textbooks now indicate the desirability of using specific medical interview behaviors to obtain information from patients, although little evidence has been presented. The results of the present study reveal a positive relationship between several specific medical interview behaviors (facilitation, the open-to-closed cone, and summarization) and the amount of information obtained from medical interviews in a real clinical practice setting.

Other studies have found a positive relationship between the use of open-ended questions and the amount of information obtained, although the present study did not produce such results (Rutter and Cox 1981; Cox et al. 1981a, b; Beckman and Frankel 1984; Roter and Hall 1987; Maguire et al. 1996; Marvel et al. 1999). Indeed, one of the authors (Y. T.) also previously found a positive relationship between the use of open-ended questions and the amount of information obtained dur- 
TABLE 3. Relationships between the medical interview behaviors used and the amount of information obtained.

\begin{tabular}{|c|c|c|c|c|c|c|c|}
\hline \multirow{2}{*}{$\begin{array}{l}\text { Medical interview } \\
\text { behaviors }\end{array}$} & \multirow{2}{*}{$\begin{array}{l}\text { Information } \\
\text { obtained* }\end{array}$} & \multicolumn{2}{|c|}{ Relationship $^{* *}$} & \multicolumn{2}{|c|}{$\begin{array}{l}\text { Relationship adjusted } \\
\text { for time duration }{ }^{* * *}\end{array}$} & \multicolumn{2}{|c|}{$\begin{array}{l}\text { Relationship adjusted for } \\
\text { other behaviors used }^{* * *}\end{array}$} \\
\hline & & $F$ & $p$ & $F$ & $p$ & $F$ & $p$ \\
\hline Open-ended questions & & 0.24 & 0.62 & 0.25 & 0.62 & 0.32 & 0.57 \\
\hline Used & 7.14 & & & & & & \\
\hline Not used & 7.23 & & & & & & \\
\hline Facilitation & & 14.9 & $<0.0001$ & 15.3 & $<0.0001$ & 18.2 & $<0.0001$ \\
\hline Frequently used & 7.66 & & & & & & \\
\hline Moderately used & 6.96 & & & & & & \\
\hline Not used & 5.91 & & & & & & \\
\hline$p$ for trend $d^{* * * *}$ & $<0.0001$ & & & & & & \\
\hline Open-to-closed cone & & 38.7 & $<0.0001$ & 40.1 & $<0.0001$ & 41.4 & $<0.0001$ \\
\hline Frequently used & 7.65 & & & & & & \\
\hline Moderately used & 6.32 & & & & & & \\
\hline Not used & 4.50 & & & & & & \\
\hline$p$ for trend $d^{* * * *}$ & $<0.0001$ & & & & & & \\
\hline Summarization & & 5.41 & 0.021 & 5.57 & 0.019 & 7.08 & 0.0082 \\
\hline Used & 7.43 & & & & & & \\
\hline Not used & 7.00 & & & & & & \\
\hline Surveying problems & & 0.91 & 0.34 & 0.94 & 0.33 & 1.21 & 0.27 \\
\hline Used & 7.23 & & & & & & \\
\hline Not used & 7.05 & & & & & & \\
\hline
\end{tabular}

${ }^{*}$ Information: duration + frequency + onset + location + quality + severity + context + modifying factors + associated symptoms.

*** ANOVA was employed.

**** The General Linear Model was applied to adjust for time duration of medical interview or the other interview behaviors used.

***** Trend was based on a simple linear regression model with scores of 0-2 assigned to the three levels.

ing 5-min medical interviews in the Objective Structured Clinical Examination (OSCE), using standardized patients (Takemura et al. 2004). One explanation might be that patients in a real practice setting do not mention all of their complaints in reply to a physician's open-ended question, but tell only what they want to tell. Another reason for the positive relationship between open-ended questions and the amount of information obtained in the author's previous study in the setting of OSCE may be that standardized patients know most of what to say beforehand, and they are ready to reply to the interviewer. It is also possible that new patients may hesitate to talk freely or may not be able to easily describe their own complaints, even if the interviewer allows them to do so using open-ended questions. Since the present study focused on information regarding physical symptoms rather than psychological or mental complaints, several closed-ended questions might be a more efficient way to obtain this kind of information than open-ended questions. This 
might be the reason why there is a positive relationship between the open-to-closed cone and the amount of information in this study.

The present study indicates no significant relationship between the interviewer's use of surveying problems and the amount of information obtained. This result conflicts with current beliefs about the utility of this behavior. The reason for this lack of relationship could be that since medical interviewers tend to use surveying at the end of the medical interview, often just after summarization, patients have already told most of what they can tell at that time and the interviewer cannot get more information, despite using surveying problems. Another possibility is that the interviewers using surveying problems may have obtained information regarding other symptoms or mental complaints that were not evaluated in this study, even though they did not get information regarding other aspects of the chief physical complaint.

There are several advantages to the present research. First, real patients were examined in a real clinical practice setting, meaning that the results of this study can more easily be generalized to daily clinical practice. Second, the number of subjects in this study was relatively large compared with previous studies, thus increasing the power of analysis of the study. A third strength of the study is the very rich dataset of videotaped interviews, which increases the reliability of the measurement. Fourth, the study resulted in an instrument (the TMIRS) that can be used to quantify medical interview behaviors as well as the information obtained from patients. Furthermore, the reliability of the TMIRS was secured in this study, where other previous studies have been weak from this standpoint. Finally, important confounding factors such as the time duration of the medical interview and other medical interview behaviors were obtained in this study, which was not always the case in previous reports. Adjustment for confounding factors helps to clarify the independent relationship between specific medical interview behaviors and the amount of information obtained.

On the other hand, there are several disad- vantages to the present study. One is that there may be more confounders for which the data should be adjusted. For example, if a patient is talkative, the interviewer might get more information from the patient. If an interviewer's personality is particularly accepting or nurturing, the patient might give information to the interviewer regardless of the interviewer's medical interview behaviors. Furthermore, since our previous study revealed a positive relationship between patient satisfaction and the amount of information obtained from patients, we might need to adjust this relationship according to patient satisfaction (Takemura et al. 2006a, b). Another disadvantage of this study is that only nine aspects of the chief physical complaint were used to determine the amount of information obtained in the medical interview. We did not evaluate whether the interviewers obtained information regarding symptoms not related to the chief complaint, information related to psychological complaints, or values and goals regarding treatment, which could lead to better decision making and improved patient rapport. Further research is needed to clarify these relationships.

\section{Acknowledgments}

We are indebted to the patients who gave up their valuable time to participate in this study. Furthermore, we wish to express our gratitude to the five experts in the teaching of medical interview skills and patient-physician communication for their significant contributions to this study, particularly the time they spent using the Delphi method to validate the medical interview rating scale. We also thank Dr. Karl T. Rew (Department of Family Medicine, University of Michigan, Ann Arbor, USA) for his assistance in English editing. Finally, we are thankful to the medical students of Mie University School of Medicine, and the residents, faculty and staff of the Department of Family Medicine, Mie University School of Medicine, for their cooperation with this study.

\section{References}

Barrier, P.A., Li, J.T.C. \& Jensen, N.M. (2003) Two words to improve physician-patient communication: what else? Mayo Clin. Proc., 78, 211-214.

Beckman, H. \& Frankel, R.M. (1984) The effect of physician behavior on the collection of data. Ann. Intern. Med., 101, 692-696. 
Beisecker, A.E. (1989) The influence of a companion on the doctor-elderly patient interaction. Health Commun., 1, 55-70.

Bird, J. \& Cohen-Cole, S.A. (1990) The three-function model of the medical interview. In: Methods in Teaching Consultation-Liaison Psychiatry, edited by M.S. Hale, Adv. Psychosom. Med., 20, 65-88.

Cox, A., Hopkinson, K. \& Rutter, M. (1981a) Psychiatric interviewing techniques. II. Naturalistic study. Br. J. Psychiatry, 138, 283-291.

Cox, A., Rutter, M. \& Holbrook, D. (1981b) Psychiatric interviewing techniques. V. Experimental study. Br. J. Psychiat., 139, 29-37.

Egan, G. (1990) The Skilled Helper: A Systematic Approach to Effective Helping. Grove, Brooks/Cole.

Hampton, J.R., Harrison, M.J.G., Mitchell, J.R.A., Mitchell, J.R., Prichard, J.S. \& Seymour, C. (1975) Relative contribution of history-taking, physical examination and laboratory investigation to diagnosis and management of medical out-patients. BMJ, 2, 486-489.

Ishikawa, H., Hashimoto, H., Roter, D.L., Yamazaki, Y., Takayama, T. and Yano, E. (2005) Patient contribution to the medical dialogue and perceived patient-centeredness. An observational study in Japanese geriatric consultations. J. Gen. Intern. Med., 20, 906-910.

Kassirer, J.P. (1983) Teaching clinical medicine by iterative hypothesis testing. N. Engl. J. Med., 309, 921-923.

Lipkins, M., Jr., Putnam, S.M. \& Lazare, A. (1995) The Medical Interview: Clinical Care, Education, and Research. New York, Springer-Verlag.

Maguire, P., Faulkner, A., Booth, K., Elliott, C. \& Hillier, V. (1996) Helping cancer patients disclose their concern. Eur. J. Cancer, 32A, 78-81.

Marvel, M.K., Epstein, R.M., Flowers, K. \& Beckman, H.B. (1999) Soliciting the patient's agenda: have we impressed? JAMA, 281, 281-287.

Participants in the Bayer-Fetzer Conference on PhysicianPatient Communication in Medical Education (2001) Essential elements of communication in medical encounters: the Kalamazoo consensus statement. Acad. Med., 76, 390-393.

Peterson, M.C., Holbrook, J.H., Von, Hales, D., Smith, N.L. \&
Staler, L.V. (1992) Contribution of the history, physical examination and laboratory investigation in making medical diagnosis. West J. Med., 156, 163-165.

Rogers, C.R. (1980) A way of being. Boston, Houghton Mifflin.

Roter, D.L. (1991) The Roter Method of Interaction Process Analysis. RIAS manual., Baltimore, Johns Hopkins University.

Roter, D.L. \& Hall, J.A. (1987) Physicians' interview styles and medical information obtained from patients. J. Gen. Intern. Med., 2, 325-329.

Rutter, M. \& Cox, A. (1981) Psychiatric interviewing techniques. I. Methods and measures. Br. J. Psychiat., 138, 273-282.

Sandler, G. (1980) The importance of the history in the medical clinic and the cost of unnecessary tests. Am. Heart J., 100, 928-931.

SAS Institute, Inc. (2004) SAS/STAT 9.1 User's Guide, Volumes 1-7. Cary, NC, SAS Institute, Inc.

Takemura, Y., Sakurai, Y., Yokoya, S., Otaki, J., Matsuoka, T., Ban, N., Hirata, I., Miki, T. \& Tsuda, T. (2004) Openended questions: are they really beneficial for gathering medical information from patients? Tohoku J. Exp. Med., 206, 151-154.

Takemura, Y., Liu, J., Atsumi, R. \& Tsuda, T. (2006a) Development of a questionnaire to evaluate patient satisfaction with medical encounters. Tohoku J. Exp. Med., 210, 373-381.

Takemura, Y., Sakurai, Y., Yokoya, S., Otaki, J., Matsuoka, T., Ban, N., Hirata, I., Miki, T. \& Tsuda, T. (2006b) Is the amount of information elicited from patients during a medical interview associated with patients' likelihood of continuing care with their physicians? Primary Care Japan, $\mathbf{4}$, 40-46.

White, J., Levison, W. \& Roter, D. (1994) "Oh, by the way"the closing moments of the medical interview. J. Gen. Intern. Med., 9, 24-28.

Wilson, A. \& Child, S. (2002) The relationship between consultation length, process and outcomes in general practice: a systematic review. Br. J. Gen. Pract., 52, 1012-1020.

Wissow, L.S., Roter, D.L. \& Wilson, M.E.H. (1994) Pediatrician interview style and mothers' disclosure of psychosocial issues. Pediatrics, 93, 289-295. 